\title{
Goodness-of-fit test of the stratified mark-specific proportional hazards model with continuous mark
}

\author{
Yanqing Sun* \\ Department of Mathematics and Statistics \\ University of North Carolina at Charlotte, Charlotte, NC 28223 \\ Mei Li \\ School of Public Health \\ Zhejiang University, Hangzhou, China \\ Peter B. Gilbert \\ Department of Biostatistics, University of Washington \\ and Fred Hutchinson Cancer Research Center, Seattle, WA 98109
}

\begin{abstract}
Motivated by the need to assess HIV vaccine efficacy, previous studies proposed an extension of the discrete competing risks proportional hazards model, in which the cause of failure is replaced by a continuous mark only observed at the failure time. However the model assumptions may fail in several ways, and no diagnostic testing procedure for this situation has been proposed. A goodness-of-fit test procedure for the stratified markspecific proportional hazards model in which the regression parameters depend nonparametrically on the mark and the baseline hazards depends nonparametrically on both time
\end{abstract}

Corresponding author's email: yasun@uncc.edu 
and the mark is proposed. The test statistics are constructed based on the weighted cumulative mark-specific martingale residuals. The critical values of the proposed test statistics are approximated using the Gaussian multiplier method. The performance of the proposed tests are examined extensively in simulations for a variety of the models under the null hypothesis and under different types of alternative models. An analysis of the 'Step' HIV vaccine efficacy trial using the proposed method is presented. The analysis suggests that the HIV vaccine candidate may increase susceptibility to HIV acquisition.

Some key words: Asymptotics; Bandwidth selection; Competing risks; Cumulative markspecific martingale residuals; Gaussian multiplier method; HIV vaccine efficacy trial; Immune responses; Local partial likelihood estimation; Mark-specific hazard ratio; Semiparametric failure time model; HVTN 502 Step trial.

Running title: Goodness-of-fit of the mark-specific proportional hazards model.

\section{Introduction}

In preventive HIV vaccine efficacy trials, the study cohort is exposed to many genetic types of circulating HIVs but the vaccine only contains HIV antigens based on one or a few types, and the vaccine protection is likely to be lower against the infecting types that are not in the vaccine construct (Gilbert et al., 1999). The similarity/dissimilarity between two HIV viruses can be measured by the genetic distance or 'mark' defined as the weighted percent mismatch of amino acids between two aligned HIV sequences. Since this distance may be unique for all infected subjects, it is natural to consider it as a continuous mark variable. A partially efficacious vaccine would likely provide better protection against infecting HIV types close to the type(s) in the vaccine construct than against infecting HIV types with greater genetic distance. Inferences about how vaccine protection varies with the mark influences the iterative development of efficacious vaccines.

Because the mark is only available for trial participants who become infected with HIV, Gilbert, McKeague and Sun (2004) investigated this problem under a competing risks model, 
where the cause of failure, a term used in the classical competing risks model, is replaced by the continuous mark. Gilbert, McKeague and Sun (2004) developed statistical methods to evaluate the dependence of the mark-specific hazard rate on the mark defined as the divergence of infecting HIV types from an HIV type(s) contained in the vaccine. Also for a continuous mark variable, Gilbert, McKeague and Sun (2008) developed statistical methods for assessing mark-specific HIV vaccine efficacy. Sun, Gilbert and McKeague (2009) extended this work to assess continuous mark-specific HIV vaccine efficacy adjusting for covariate effects. The methods were applied to analyze the first HIV vaccine efficacy trial conducted by VaxGen Inc. from 1998 to 2003 in North America and the Netherlands.

The competing risks model with continuous marks also has applications in other situations. In the development of flu vaccines, the endpoint is clinically significant infection with flu and the mark of interest is the genetic distance between the infecting flu virus and the virus represented in the vaccine. It is well known for flu vaccines that moderate genetic mismatch between an exposing flu virus and the virus represented in the vaccine causes vaccine failure, which has necessitated development of a new vaccine each year that is closely matched to the contemporary circulating flu strains. In studies of quality-adjusted lifetime (Olschewski and Schumacher, 1990), the lifetime medical cost is a mark variable measured at death. In another example (Huang and Louis, 1998), the endpoint is onset of a disease and the mark is the incubation period between infection and disease onset. Recent competing risks failure time research in CSDA includes Han and Balakrishnan (2010), Cramer and Schmiedt (2011), and Feizjavadian and Hashemi (in press).

Let $T$ be the failure time, $V$ a continuous mark variable, and $Z(t)$ a time-dependent $p$ dimensional covariate. The conditional mark-specific hazard function is defined as $\lambda(t, v \mid z)=$ $f(t, v \mid Z(t)=z) / S(t \mid Z(t)=z)$, where $f(t, v \mid Z(t)=z)$ is the conditional density of $(T, V)$ at $(t, v)$ given $Z(t)=z$ and $S(t \mid Z(t)=z)$ is the conditional survival function of $T$ at $t$ given $Z(t)=z$. Let $\lambda_{k}(t, v \mid z)$ be the conditional mark-specific hazard function at time $t$ with mark $v$ for a subject in stratum $k$ with covariate $z$. We assume that the mark $V$ takes value in the interval $[0,1]$, rescaled if necessary. The stratified mark-specific proportional hazards (PH) 
model posits that

$$
\lambda_{k}(t, v \mid z)=\lambda_{0 k}(t, v) \exp \left\{\beta^{T}(v) z(t)\right\}, \quad \text { for } t \geq 0,0 \leq v \leq 1
$$

for $k=1,2, \ldots, K$, where $K$ is the number of the strata, $\lambda_{0 k}(t, v)$ is an unspecified baseline mark-specific hazard function for stratum $k, z(t)$ is a $p$-dimensional possibly time-dependent covariate and $\beta(v)$ is a $p$-dimensional vector of regression functions.

Sun, Gilbert and McKeague (2009) developed an estimation method for model (1) when $K=1$. In practice, different key subgroups (e.g., men and women; subjects living in different geographic regions) typically have different baseline mark-specific hazards of HIV infection. Model (1) is a generalization of the mark PH model of Sun, Gilbert, and McKeague (2009), allowing different baseline functions for different strata. Sun and Gilbert (2012) and Gilbert and Sun (2014) developed estimation and hypothesis testing procedures respectively for assessing mark-specific vaccine efficacy defined as one minus the mark-specific hazard ratio (vaccine/placebo) of infection. The methods were used to analyze the RV144 HIV vaccine efficacy trial in Thailand, which was the first trial to demonstrate partial efficacy of an HIV vaccine (Rerks-Ngarm et al., 2009).

However, the usefulness of the statistical procedures for model (1) relies on the validity of the model. The model (1) may fail in three ways: (i) the time invariance of the hazard ratio does not hold; (ii) the exponential form of the link function for the hazard ratio is inappropriate; (iii) the functional forms of individual covariates in the exponent of the model are misspecified. The model misspecification can have detrimental effects on the validity and efficiency of the partial likelihood inference for the proportional hazards model (Lagakos and Schoenfeld, 1984; Struthers and Kalbfleisch, 1986; Lagakos, 1988(b); Lin and Wei, 1989).

This paper develops a goodness-of-fit testing procedure for the stratified mark-specific $\mathrm{PH}$ model with continuous marks. Following the development of the goodness-of-fit tests of Lin, Wei and Ying (1993) for the Cox model, the proposed test statistics are constructed based on weighted cumulative mark-specific martingale residual processes. However, the theoretical development of the goodness-of-fit tests for model (1) is more challenging because of the nonparametric regression function $\beta(v)$ of the model. The weak convergence of the weighted 
mark-specific martingale residual processes is investigated. The asymptotic distributions of the proposed test statistics are derived. The performance of the proposed tests is examined extensively in simulations for a variety of the models under the null hypothesis and under different types of alternative models. The simulation study shows that the proposed goodnessof-fit test procedure works well. We present an analysis of the 'Step' HIV vaccine efficacy trial using the stratified mark-specific proportional hazards model and the model checking is conducted using the proposed method. Our analysis suggests that the the Merck Adenovirus 5 vaccine may increase susceptibility to HIV acquisition.

The rest of the paper is organized as follows. The goodness-of-fit test procedure for the stratified mark-specific PH model is presented in Section 2. A simulation study is conducted to examine the finite sample performance of the proposed method in Section 3. The proposed method is applied to the Step trial in Section 4. Proofs of the main results are placed in the online Supplementary Material.

\section{Goodness-of-fit test}

Let $n_{k}$ be the number of observations in the $k$ th stratum. For an individual in the $k$ th stratum, let $T_{k}$ be the failure time, $V_{k}$ the mark observed at the time of failure with support on $[0,1]$, and $Z_{k}(\cdot)=\left\{Z_{k}(t), 0 \leq t \leq \tau\right\}$ the associated $p$-dimensional covariate process. Suppose that $\left(T_{k}, V_{k}, Z_{k}(t)\right)$ follows model (1). Under right censoring, the observed random variables are $\left(X_{k}, \delta_{k}, \delta_{k} V_{k}, Z_{k}(t)\right)$, where $X_{k}=\min \left(T_{k}, C_{k}\right), \delta_{k}=I\left(T_{k} \leq C_{k}\right)$, and $C_{k}$ is the censoring random variable. We assume that $T_{k}$ and $V_{k}$ are independent conditional on $Z_{k}(\cdot)$. The mark variable $V_{k}$ is observed only if the failure time is uncensored. The right censored markspecific failure time data, $\left(X_{k i}, \delta_{k i}, \delta_{k i} V_{k i}, Z_{k i}(\cdot)\right), i=1, \ldots, n_{k}$, are independent identically distributed (iid) replicates of $\left(X_{k}, \delta_{k}, \delta_{k} V_{k}, Z_{k}(\cdot)\right)$ from stratum $k$, for $k=1,2, \ldots, K$. Let $\tau$ be the end of the follow-up time. All failure times beyond $\tau$ are considered censored.

The development of the goodness-of-fit test procedure for model (1) relies on the model estimation, which is the basis of the martingale residuals defined below under the assumed 
model. For the $i$ th individual in stratum $k$, let $N_{k i}(t, v)=I\left(X_{k i} \leq t, V_{k i} \leq v, \delta_{k i}=1\right)$ be the marked counting process with jump at the uncensored failure time $T_{k i}$ with $V_{k i}$ the associated mark. Let $Y_{k i}(t)=I\left(X_{k i} \geq t\right)$ be the at-risk process. Based on Sun and Gilbert (2012), the localized version of the $\log$-partial likelihood function for $\beta=\beta(v)$ at a fixed $v$ is given by

$$
l(v, \beta)=\sum_{k=1}^{K} \sum_{i=1}^{n_{k}} \int_{0}^{1} \int_{0}^{\tau} K_{h}(u-v)\left[\beta^{T} Z_{k i}(t)-\log \left(\sum_{j=1}^{n_{k}} Y_{k j}(t) e^{\beta^{T} Z_{k j}(t)}\right)\right] N_{k i}(d t, d u),
$$

where $K_{h}(x)=K(x / h) / h, K(\cdot)$ is a symmetric kernel function with support $[-1,1]$ and $h=h_{n}$ is the bandwidth. The maximum partial likelihood estimator (MPLE) $\hat{\beta}(v)$ for $\beta(v)$ is obtained by maximizing $l(v, \beta)$. Taking the derivative of $l(v, \beta)$ with respect to $\beta$, the score function can be written as

$$
U(v, \beta)=\sum_{k=1}^{K} \sum_{i=1}^{n_{k}} \int_{0}^{1} \int_{0}^{\tau} K_{h}(u-v)\left[Z_{k i}(t)-\frac{S_{k}^{(1)}(t, \beta)}{S_{k}^{(0)}(t, \beta)}\right] N_{k i}(d t, d u),
$$

where $S_{k}^{(j)}(t, \beta)=n_{k}^{-1} \sum_{i=1}^{n_{k}} Y_{k i}(t) \exp \left\{\beta^{T} Z_{k i}(t)\right\} Z_{k i}(t)^{\otimes j}$ for $j=0,1,2$. Here $z \in \mathbb{R}^{p}$, $z^{\otimes 0}=1, z^{\otimes 1}=z$ and $z^{\otimes 2}=z z^{T}$. The MPLE $\hat{\beta}(v)$ is the solution to $U(v, \hat{\beta}(v))=0$, which can be obtained using the Newton-Raphson algorithm. The asymptotic variance of $(n h)^{1 / 2}(\hat{\beta}(v)-\beta(v))$ can be estimated by $\nu_{0}(\hat{\Sigma}(v))^{-1}$, where $\nu_{0}=\int K^{2}(u) d u$ and $\hat{\Sigma}(v)=$ $-l_{\beta}^{\prime \prime}(v, \hat{\beta}(v)) / n$. It is easy to check that $\nu_{0}=3 / 5$ for Epanechnikov's kernel $K(x)=\frac{3}{4}(1-$ $\left.x^{2}\right),-1<x<1$.

The baseline function $\lambda_{0 k}(t, v)$ can be estimated by smoothing the increments of the following estimator of the doubly cumulative mark-specific baseline hazard function $\Lambda_{0 k}(t, v)=$ $\int_{0}^{t} \int_{0}^{v} \lambda_{0 k}(s, u) d s d u$ :

$$
\hat{\Lambda}_{0 k}(t, v)=\int_{0}^{t} \int_{0}^{v} \frac{N_{k \cdot}(d s, d u)}{n_{k} S_{k}^{(0)}(s, \hat{\beta}(u))}
$$

where $N_{k} .(t, v)=\sum_{i=1}^{n_{k}} N_{k i}(t, v)$. A kernel estimator of $\lambda_{0 k}(t, v)$ can be obtained using $\hat{\lambda}_{0 k}(t, v)=\int_{0}^{\tau} \int_{0}^{1} K_{h_{1}}^{(1)}(t-s) K_{h_{2}}^{(1)}(v-u) \hat{\Lambda}_{0 k}(d s, d u)$, where $K_{h_{1}}^{(1)}(x)=K^{(1)}\left(x / h_{1}\right) / h_{1}$ and $K_{h_{2}}^{(2)}(x)=K^{(2)}\left(x / h_{2}\right) / h_{2}, K^{(1)}(\cdot)$ and $K^{(2)}(\cdot)$ are kernel functions, and $h_{1}$ and $h_{2}$ are the corresponding bandwidths. 
Let $M_{k i}(t, v)=\int_{0}^{t} \int_{a}^{v}\left\{N_{k i}(d s, d u)-Y_{k i}(s) \lambda_{k}\left(s, u \mid Z_{k i}(s)\right) d s d u\right\}$, where $0<a<1$ is a number close to zero to avoid the boundary problem in the nonparametric estimation of $\beta(v)$ for $v$ near zero. According to Aalen and Johansen (1978), $M_{k i}\left(\cdot, v_{1}\right)$ and $M_{k i}\left(\cdot, v_{2}\right)-$ $M_{k i}\left(\cdot, v_{1}\right)$ are orthogonal square integrable martingales with respect to $\mathcal{F}_{t}$ for any $0 \leq a \leq$ $v_{1} \leq v_{2} \leq 1$, where $\mathcal{F}_{t}=\sigma\left\{I\left(X_{k i} \leq s, \delta_{k i}=1\right), I\left(X_{k i} \leq s, \delta_{k i}=0\right), V_{k i}\left(X_{k i} \leq s, \delta_{k i}=\right.\right.$ 1), $\left.Z_{k i}(s) ; 0 \leq s \leq t, k=1, \ldots, K, i=1, \ldots, n_{k}\right\}$ is the right-continuous filtration generated by $\left\{N_{k i}(s, v), Y_{k i}(s), Z_{k i}(s) ; 0 \leq s \leq t, 0 \leq v \leq 1, k=1, \ldots, K, i=1, \ldots, n_{k}\right\}$. The process $M_{k i}(t, v)$ is the integration of the mark-specific martingale, which we refer to as the cumulative mark-specific martingale. It follows from Sun, Gilbert and McKeague (2009) that $M_{k i}(t, v)$ is a martingale with respect to $\mathcal{F}_{t}$ for each fixed $v$.

We derive the model checking test statistics based on the cumulative mark-specific martingale residuals defined as

$$
\hat{M}_{k i}(t, v)=\int_{0}^{t} \int_{a}^{v}\left\{N_{k i}(d s, d u)-Y_{k i}(s) \exp \left((\hat{\beta}(u))^{T} Z_{k i}(s)\right) \hat{\Lambda}_{0 k}(d s, d u)\right\},
$$

where $\hat{\beta}(u)$ is the MPLE given in Section 2, and $\hat{\Lambda}_{0 k}(t, v)$ is defined in (4). $\hat{M}_{k i}(t, v)$ may be interpreted as the difference between the observed and predicted number of events before time $t$ with mark less than $v$ for the $i$ th subject in the $k$ th stratum. Thus the martingale residuals are informative about model misspecification. Similar to the result for martingale residuals in the context of the standard Cox model where the sum of all martingale residuals is exactly zero, it is easy to derive that $n^{-1 / 2} \sum_{k=1}^{K} \sum_{i=1}^{n_{k}} \hat{M}_{k i}(t, v)=o_{p}(1)$. The asymptotic term is $o_{p}(1)$ rather than exactly zero due to the local smoothing for the mark.

We construct goodness-of-fit test statistics based on the sum of weighted cumulative markspecific martingale residuals. Following the general approach of Lin, Wei and Ying (1993), we introduce the test process $W(t, v, z)=\left(\left(W_{1}(t, v, z)\right)^{T}, \ldots,\left(W_{K}(t, v, z)\right)^{T}\right)^{T}$, where

$$
W_{k}(t, v, z)=n^{-1 / 2} \sum_{i=1}^{n_{k}} \int_{0}^{t} \int_{a}^{v} g_{k}\left(Z_{k i}(s), z\right) d \hat{M}_{k i}(s, u)
$$

for $1 \leq k \leq K$, and $g_{k}\left(Z_{k i}(t), z\right)$ is an $r$-dimensional column vector of known bounded functions of $Z_{k i}(t)$ and $z$. For example, one may take $g_{k}\left(Z_{k i}(t), z\right)=f_{k}\left(Z_{k i}(t)\right) I\left(Z_{k i}(t) \leq\right.$ 
$z)$, where $f_{k}(\cdot)$ is a known function, $I\left(Z_{k i}(t) \leq z\right)=\left(I\left(Z_{1 k i}(t) \leq z_{1}\right), \ldots, I\left(Z_{p k i}(t) \leq\right.\right.$ $\left.\left.z_{p}\right)\right)^{T}$, and $Z_{j k i}(t)$ is the $j$ th element of $Z_{k i}(t)$, in which case $r=p$. If model (1) holds, the process $W(t, v, z)$ is expected to fluctuate randomly about zero. Various test statistics can be constructed by selecting different weight functions $g_{k}(\cdot, \cdot)$ and using different functionals of the process $W(t, v, z)$.

Let $W_{k}^{(j)}(t, v, z)$ be the $j$ th component of $W_{k}(t, v, z)$ corresponding to the $j$ th component $g_{k}^{(j)}\left(Z_{k i}(t), z\right)$ of $g_{k}\left(Z_{k i}(t), z\right)$ for $1 \leq j \leq r$. We investigate the following supremum test statistic for assessing goodness-of-fit of model (1):

$$
T=\sup _{1 \leq k \leq K} \sup _{(t, v, z) \in \mathcal{C}}\left\|W_{k}(t, v, z)\right\|,
$$

where $\left\|W_{k}(t, v, z)\right\|=\sum_{j=1}^{r}\left|W_{k}^{(j)}(t, v, z)\right|$ and $\mathcal{C}=[0, \tau] \times[a, b] \times \mathcal{Z}$. Here $\mathcal{Z}$ is the set of grid points in the support of the covariate $Z(\cdot)$ and $[a, b] \in(0,1)$.

We introduce three test statistics, namely $T_{s}, T_{o}$ and $T_{a}$, by selecting different functions of $g_{k}\left(Z_{k i}(t), z\right)$ in (7). The statistic $T_{s}$ is obtained by choosing $g_{k}\left(Z_{k i}(t), z\right)=Z_{k i}(t)$, which is the supremum test based on the score process. The test $T_{s}$ can be used to test for the proportionality of the mark-specific hazards. The test statistic $T_{o}$ is obtained by setting $g_{k}\left(Z_{k i}(t), z\right)=I\left(Z_{k i}(t) \leq z\right)$. The test statistic $T_{o}$ can be used to test overall fitness of the model because the supremum is taken over the product space of the follow-up time and covariates. We take the third test statistic $T_{a}=T_{s}+T_{o}$, which may be obtained by choosing $g_{k}\left(Z_{k i}(t), z\right)=\left(Z_{k i}(t)^{T}, I\left(Z_{k i}(t)^{T} \leq z^{T}\right)\right)^{T}$. The test statistic $T_{a}$ balances the power against the time-varying alternative and an arbitrary departure from model (1).

The distribution of $T$ under the null hypothesis is complicated and intractable. We show in the next section that $W(t, v, z)$ converges weakly to a mean zero Gaussian process under model (1). The asymptotic null distribution of $W(t, v, z)$ can be approximated using the Gaussian multiplier method (Lin, Wei and Ying, 1993). Thus, with the application of the continuous mapping theorem, the critical value of the test statistic $T$ can be approximated using the Gaussian multiplier method. The finite same performances of the test statistics $T_{s}, T_{o}$ and $T_{a}$ under the null hypothesis and for various departures from the model (1) are examined in Section 3. 
We study the asymptotic properties of the test process $W(t, v, z)$ on $[0, \tau] \times[a, b] \times \mathbb{R}^{p}$. Let $S_{k g}^{(0)}(t, z, \beta)=n_{k}^{-1} \sum_{i=1}^{n_{k}} Y_{k i}(t) \exp \left\{\beta^{T} Z_{k i}(t)\right\} g_{k}\left(Z_{k i}(t), z\right)$ and $S_{k g}^{(1)}(t, z, \beta)=n_{k}^{-1} \sum_{i=1}^{n_{k}} Y_{k i}(t)$ $\exp \left\{\beta^{T} Z_{k i}(t)\right\} g_{k}\left(Z_{k i}(t), z\right) Z_{k i}^{T}(t)$. The following theorem presents the weak convergence of the process $W(t, v, z)$. The regularity conditions (A.1)-(A.6) for the asymptotic results are stated in Condition A in the Appendix. The proof of Theorem 1 is placed in the online Supplementary Material.

Theorem 1. Under the conditions (A.1)-(A.6) stated in the Appendix, we have, for $1 \leq k \leq K$,

$$
\begin{aligned}
W_{k}(t, v, z)= & n^{-1 / 2} \sum_{l=1}^{K} \sum_{i=1}^{n_{l}} \int_{0}^{\tau} \int_{a}^{v} I(l=k) I(s \leq t)\left[g_{l}\left(Z_{l i}(s), z\right)-\frac{S_{l g}^{(0)}(s, z, \beta(u))}{S_{l}^{(0)}(s, \beta(u))}\right] M_{l i}(d s, d u) \\
& +n^{-1 / 2} \sum_{l=1}^{K} \sum_{i=1}^{n_{l}} \int_{0}^{\tau} \int_{a}^{v} R_{k}(t, u, z)(\Sigma(u))^{-1}\left[Z_{l i}(s)-\frac{S_{l}^{(1)}(s, \beta(u))}{S_{l}^{(0)}(s, \beta(u))}\right] M_{l i}(d s, d u) \\
& +o_{p}(1)
\end{aligned}
$$

as $n \rightarrow \infty$, where

$$
R_{k}(t, u, z)=\left(n_{k} / n\right) \int_{0}^{t}\left(\frac{S_{k g}^{(0)}(s, z, \beta(u))\left(S_{k}^{(1)}(s, \beta(u))\right)^{T}}{S_{k}^{(0)}(s, \beta(u))}-S_{k g}^{(1)}(s, z, \beta(u))\right) \lambda_{0 k}(s, u) d s .
$$

The process $W(t, v, z)=\left(\left(W_{1}(t, v, z)\right)^{T}, \ldots,\left(W_{K}(t, v, z)\right)^{T}\right)^{T}$ converges weakly to a multidimensional mean zero Gaussian random process on $[0, \tau] \times[a, b] \times \mathbb{R}^{p}$ as $n \rightarrow \infty$.

Let $\left\{\xi_{l i}, i=1, \ldots, n_{l}, l=1, \ldots, K\right\}$ be iid standard normal random variables. Using the Gaussian multiplier technique of Lin, Wei and Ying (1993), the distribution of $W(t, v, z)$ can be approximated by the distribution of $W^{*}(t, v, z)=\left(\left(W_{1}^{*}(t, v, z)\right)^{T}, \ldots,\left(W_{K}^{*}(t, v, z)\right)^{T}\right)^{T}$, where, for $1 \leq k \leq K$,

$$
\begin{aligned}
& W_{k}^{*}(t, v, z) \\
& =n^{-1 / 2} \sum_{l=1}^{K} \sum_{i=1}^{n_{l}} \int_{0}^{\tau} \int_{a}^{v} I(l=k) I(s \leq t)\left[g_{l}\left(Z_{l i}(s), z\right)-\frac{S_{l g}^{(0)}(s, z, \hat{\beta}(u))}{S_{l}^{(0)}(s, \hat{\beta}(u))}\right] \xi_{l i} N_{l i}(d s, d u) \\
& \quad+n^{-1 / 2} \sum_{l=1}^{K} \sum_{i=1}^{n_{l}} \int_{0}^{\tau} \int_{a}^{v} \hat{R}_{k}(t, u, z)(\hat{\Sigma}(u))^{-1}\left[Z_{l i}(s)-\frac{S_{l}^{(1)}(s, \hat{\beta}(u))}{S_{l}^{(0)}(s, \hat{\beta}(u))}\right] \xi_{l i} N_{l i}(d s, d u),
\end{aligned}
$$


Here,

$$
\hat{R}_{k}(t, u, z)=\left(n_{k} / n\right) \int_{0}^{t}\left(\frac{S_{k g}^{(0)}(s, z, \hat{\beta}(u))\left(S_{k}^{(1)}(s, \hat{\beta}(u))\right)^{T}}{S_{k}^{(0)}(s, \hat{\beta}(u))}-S_{k g}^{(1)}(s, z, \hat{\beta}(u))\right) d \hat{\Lambda}_{0 k, u}(s),
$$

$\hat{\Sigma}(v)=-l_{\beta}^{\prime \prime}(v, \hat{\beta}(v)) / n$ is the minus second order partial derivative of log-partial likelihood function with respect to $\beta$ divided by $n$, and

$$
\hat{\Lambda}_{0 k, u}(t)=\int_{0}^{t} \int_{0}^{1} K_{h}(u-x) \frac{d N_{k} \cdot(d s, d x)}{n_{k} S_{k}^{(0)}(s, \hat{\beta}(x))} .
$$

is the estimator of the mark-specific cumulative baseline function $\Lambda_{0 k, u}(t)=\int_{0}^{t} \lambda_{0 k}(s, u) d s$.

By Lemma 1 of Sun and Wu (2005), the distribution of $W(t, v, z)$ can be approximated by the conditional distribution of $W^{*}(t, v, z)=\left(W_{1}^{*}(t, v, z), \ldots, W_{K}^{*}(t, v, z)\right)$ given the observed data sequence. Let $W_{k}^{*(j)}(t, v, z)$ be the $j$ th component of $W_{k}^{*}(t, v, z)$. The continuous mapping theorem implies that the distribution of $T$ can be approximated by the empirical distribution of a large number of copies, say 500, of $T^{*}=\sup _{1 \leq k \leq K} \sup _{(t, v, z) \in \mathcal{C}}\left\|W_{k}^{*}(t, v, z)\right\|$, obtained through repeatedly generating independent sets of normal random variables $\left\{\xi_{l i}, i=\right.$ $\left.1, \ldots, n_{l}, l=1, \ldots, K\right\}$ while holding the observed data fixed, where $\left\|W_{k}^{*}(t, v, z)\right\|=\sum_{j=1}^{r}$ $\left|W_{k}^{*(j)}(t, v, z)\right|$. The critical value, $T_{\alpha}$, of the test statistic $T$ at significance level $\alpha$ can be approximated by the upper $\alpha$ quantile of the distribution of $T^{*}$. Goodness-of-fit of model (1) is rejected at significance level $\alpha$ if $T$ is greater than $T_{\alpha}$.

\section{Simulation study}

In this section, we conduct a simulation study to examine the finite sample performances of the proposed goodness-of-fit test statistics $T_{s}, T_{o}$ and $T_{a}$ for their empirical sizes and empirical powers against various departures from the model (1).

We use the Epanechnikov kernel $K(x)=.75\left(1-x^{2}\right) I\{|x| \leq 1\}$ for the kernel smoothing throughout. We take $\tau=2.0$ as the end of follow-up time. The bandwidth is selected using the formula $h=C \hat{\sigma}_{V} n^{-1 / 3}$, where $\hat{\sigma}_{V}$ is the estimated standard error of the observed marks for uncensored failure times and $C$ is a constant ranging from 2 to 5 . This selection of $h$ satisfies 
condition (A.5). Similar bandwidth selection has been used in Zhou and Wang (2000). We choose $C=3$ for our simulation study. We also tried $C=2$ and $C=4$, which gave similar results to those with $C=3$. In practice, a larger $C$ can be used if the distribution of the observed marks is skewed or marks are sparse in some areas. We chose $a=0.1$ and $b=0.9$ to avoid the boundary effect near the end points 0 and 1 since the bias and variance are larger at the boundaries. Theoretically, the boundary points refer to those in the intervals $[0, h)$ and $(1-h, 1]$. The estimated bandwidths for all of our simulation models are in the range between 0.2 and 0.4. Choosing $a=h$ and $b=1-h$ would make the empirical sizes of the tests more stable around the nominal level, but it may trim away too much data and thus reduce the power of the tests. We take $a=0.1$ and $b=0.9$ as a compromise between size and power. In practice, $a=h / 2$ and $b=1-h / 2$ is recommended.

The following mark-specific PH model with $K=1$ is considered to check the empirical sizes of the proposed tests:

$$
\lambda_{k}(t, v \mid z)=\lambda_{0 k}(t, v) \exp \{(\alpha-\beta(1-v)) z\}, \quad t \geq 0,0 \leq v \leq 1
$$

where the baseline function is $\lambda_{0 k}(t, v)=\exp (0.3 v)$, and the covariate $Z_{k i}$ is from a uniform distribution on $(0,1)$. The empirical sizes are evaluated under model (11) with four sets of values of $(\alpha, \beta)$. We define $M_{01}:(\alpha, \beta)=(0,0) ; M_{02}:(\alpha, \beta)=(-0.69,0) ; M_{03}:(\alpha, \beta)=$ $(0,0.6)$ and $M_{04}:(\alpha, \beta)=(0,0.8)$. The empirical sizes are also evaluated under the following model to show the versatility of the proposed tests when the regression function $\beta(v)$ is not a linear function of $v$ :

$$
M_{05}: \quad \lambda_{k}(t, v \mid z)=2 v \exp \left(0.3 v^{2}\right) \exp \left\{\left(-0.4\left(1-v^{2}\right)\right) z\right\}, \quad t \geq 0,0 \leq v \leq 1,
$$

where the covariate $Z_{k i}$ is from a uniform distribution on $(0,1)$. For each of the null models $M_{01}, M_{02}, M_{03}, M_{04}$ and $M_{05}$, the random right censoring times are generated from an exponential distribution, independent of $\left(T_{k i}, V_{k i}\right)$, and yield around $30 \%$ censoring.

The empirical sizes are calculated based on 500 simulations and 500 Gaussian multiplier samples. Table 1 shows the empirical sizes of the tests under the null models $M_{01}$ to $M_{05}$ for sample sizes of $n=300$ and 400 . The empirical sizes for the test statistics $T_{o}$ and $T_{a}$ are close 
to the 0.05 nominal level for $n=300$ and 400 . The empirical sizes for the test statistic $T_{s}$ is slightly larger for $n=300$, but it becomes closer to the 0.05 nominal level as the sample size increases to 400 .

[Table 1 about here]

The powers of the test statistics are examined for the three types of alternatives: (i) Time varying alternative where the mark-specific hazard ratio is time varying; (ii) Link function alternative where the exponential form of the link function for the mark-specific hazard ratio is inappropriate; (iii) Covariate form alternative where the functional forms of individual covariates in the exponent of the model are misspecified. In the following model specifications, the covariate $Z$ follows a uniform distribution on $(0,1)$.

The empirical powers of the proposed tests against the time-varying alternative model are evaluated under the following model:

$$
\lambda(t, v \mid z)=\lambda_{0}(t, v) \exp \{[\theta \log t-0.1(1-v)] z\}, \quad 0<v<1, t>0
$$

where $\lambda_{0}(t, v)=\exp \{\log t+0.3 v\}$. We consider three models corresponding to three different values of $\theta$ under model (13), defined as $M_{11}: \theta=2.0 ; M_{12}: \theta=2.5$; and $M_{13}: \theta=3.0$. As $\theta$ increases, the rate of change with respect to $t$ of the relative mark-specific hazard ratio increases, representing increasing departures from the null hypothesis.

The empirical powers of the proposed tests against the link function alternative model are evaluated under the following model:

$$
\lambda(t, v \mid z)=0.05+10(1-v)^{\alpha} z
$$

We consider three models corresponding to three different values of $\alpha$ under model (14), defined as $M_{21}: \alpha=0.15 ; M_{22}: \alpha=0.20$; and $M_{23}: \alpha=0.25$.

The empirical powers of the proposed tests against the misspecified covariate form are evaluated under the following model:

$$
\lambda(t, v \mid z)=\lambda_{0}(t, v) \exp \left\{-3.2 \gamma(1-v)(z-0.5)^{2}\right\}
$$


where $\lambda_{0}(t, v)=e^{0.3 v}$. We consider three models corresponding to three different values of $\gamma$ under model (15), defined as $M_{31}: \gamma=3.5 ; M_{32}: \gamma=4.0$; and $M_{33}: \gamma=4.5$.

For each of the models that are used for evaluating the powers of the tests, the random right censoring times are generated from an exponential distribution, independent of $\left(T_{k i}, V_{k i}\right)$, and

yield around $30 \%$ censoring. The bandwidth is selected using the formula $h=3 \hat{\sigma}_{V} n^{-1 / 3}$ as with the scenarios for evaluating the sizes of the tests.

The empirical powers of the tests at significance level 0.05 for sample sizes $n=300$ and 400 are given in Table 2. Each entry of the table is based on 500 simulation runs and 500 Gaussian multiplier samples. Table 2 shows that the test $T_{s}$ is more powerful than the tests $T_{o}$ and $T_{a}$ against the time varying alternative where the mark-specific hazard ratio is time varying. The test $T_{o}$ is more powerful against the alternative where the exponential form of the link function for the mark-specific hazard ratio is inappropriate. It is also more powerful against the alternative where the functional form of the covariate in the exponent of the model is misspecified. The test $T_{s}$ has very poor power against the last two types of alternatives. The test $T_{a}$ has the second best power against all three types of alternatives. The powers of the tests increase as the departure increases. The powers of all three tests increase with the sample size except for the test $T_{s}$ for the alternative where the functional form of the covariate is misspecified, in which case $T_{s}$ has basically no power.

[Table 2 about here]

\section{Application to a vaccine efficacy trial}

The 'Step' trial was a preventive HIV vaccine efficacy trial conducted in North and South America and Australia from 2005 to 2007 (Buchbinder et al., 2008). The trial randomized 1836 HIV negative men to receive either the Merck Adenovirus 5 (Ad5) vaccine (MRKAd5) or placebo, of whom 53 and 34 subjects experienced the primary endpoint of HIV infection during the 3 year follow-up period, respectively. The randomization was stratified by whether 
a volunteer had pre-existing immunity to the Ad5 vector that was used in the vaccine, defined by an Ad5 neutralization titer greater than 200. One thousand and fifty-eight volunteers had Ad5 titer below 200 and 778 volunteers had Ad5 titer above 200.

The sequencing labs attempted to derive HIV sequences via single-genome-amplification from all 87 HIV infected subjects, and were successful for 65. For those with Ad5 titer $\leq 200$ there were 54 total infections: 29 of 522 in the vaccine group (with 7 missing marks) and 25 of 536 in the placebo group (with 8 missing marks), with annual HIV incidences of $5.6 \%$ and $4.7 \%$. For the Ad5 titer $>200$ subgroup there were 33 total infections: 24 of 392 in the vaccine group (with 7 missing marks) and 9 of 386 in the placebo group (with 0 missing marks), with annual HIV incidences $6.1 \%$ and $2.3 \%$. We analyze the data excluding the 22 HIV infected men with no sequence data.

Three HIV-1 genes, gag, pol, and nef, are contained in the MRKAd5 vaccine, which stimulates immune responses to the expressed proteins Gag, Pol, and Nef that could plausibly make the vaccine effect on infection vary with genetic distance to the protein. Genetic distances were computed for each of the proteins Gag, Pol, and Nef as well as for Gag-Pol-Nef combined and for all HIV-1 proteins not represented in the vaccine combined, Env-Rev-TatVif-Vpr-Vpu, which serves as a control. The Gag, Pol, Nef, and Gag-Pol-Nef distances use the vaccine-construct sequences as the reference strain and the Env-Rev-Tat-Vif-Vpr-Vpu distances use the central HXB2 reference strain as the reference strain. Because the vaccine should not be able to stimulate immune responses to proteins excluded from the vaccine, it is expected that the vaccine effect on infection would not vary with this control genetic distance. In addition, the genetic distances were calculated for each infected subject based on two different bioinformatics methods: NetMHC (Buus, et al., 2003) and Epipred (Heckerman, Kadie, and Listgarten, 2007). Details on these methods for calculating the genetic distances can be found in Sun, Li and Gilbert (2013). Thus each mark is identified by three components: the bioinformatics method (Method), the reference strain (Ref) and the Protein. Method refers to the bioinformatics method (NetMHC or Epipred), Ref is for the reference HIV strain (the MRKAd5 vaccine sequence or the central HXB2 strain), and Protein is one of the five HIV-1 
proteins/sets of proteins (Gag, Pol, Nef, Gag-Pol-Nef, Env-Rev-Tat-Vif-Vpr-Vpu). Consequently, each mark is denoted using the form Method-Ref-Protein.

We fit the stratified mark-specific PH model

$$
\lambda_{k}(t, v \mid z)=\lambda_{0 k}(t, v) \exp \{\beta(v) z\},
$$

for $k=1$ and 2, where the covariate $z$ is the treatment indicator, with $z=1$ for the vaccine group and $z=0$ for the placebo group, and the two strata are the Ad5 $\leq 200$ and Ad5 $>200$ titer groups. The maximum of the observed or censored failure times is 1345 days. We set $\tau=735$ days, which is the maximum of the observed failure times. The ten different mark variables are standardized to the interval $[0,1]$ by subtracting the minimum and dividing by the range of the marks. In addition, before the standardization to the interval $[0,1]$, the logarithm transformation $\log (\operatorname{mark}+1)$ was applied to the marks NetMHC-MRKAd5-Gag and NetMHC-MRKAd5-Gag-Pol-Nef, and the transformation $\log (\operatorname{mark}+0.01)$ was applied to the marks Epipred-MRKAd5-Pol and Epipred-MRKAd5-Gag-Pol-Nef to make the distributions of the observed marks less skewed and less sparse in the right tails.

The estimated mark-specific hazard ratio $\exp \{\hat{\beta}(v)\}$ of the vaccine versus placebo group against the mark $v$ along with its $95 \%$ pointwise confidence intervals for each of the ten marks are plotted in Figure 1. For each mark, the bandwidth is estimated using the formula $h=$ $5 \hat{\sigma}_{V} n^{-1 / 3}$. The $95 \%$ confidence intervals are calculated based on the variance estimator of Sun and Gilbert (2012). The plots are limited to the interval $[0,0.8]$ because of the sparsity of data points with marks near 1.0.

[Figure 1 about here]

Figure 1 shows that the curves of the estimated mark-specific hazard ratios (vaccine vs. placebo) are above one for many of the ten marks. There is an upward trend in the curves of the estimated mark-specific hazard ratios as the mark increases for Epipred-MRKAd5Gag, NetMHC-MRKAd5-Gag, Epipred-MRKAd5-Nef, Epipred-MRKAd5-Gag-Pol-Nef and NetMHC-MRKAd5-Gag-Pol-Nef. The plots suggest that the vaccine may increase susceptibility to HIV acquisition for HIV strains that are quite distant from the vaccine strains. 
However, we also notice that the hazard ratios are less than 1 for NetMHC-MRKAd5-Gag and Epipred-MRKAd5-Gag-Pol-Nef when the marks are less than 0.32. They are also less than 1 for NetMHC-MRKAd5-Gag-Pol-Nef when the marks are less than 0.27. Since all the confidence intervals are quite wide and include the horizontal line of hazard ratio at 1, the perceived higher mark-specific hazard in the vaccine group over the placebo group is not statistically significant. The upward pattern of the curves for the estimated mark-specific hazard ratios in some cases is intriguing, generating hypotheses for future research. However, caution is warranted in drawing conclusions because of potential bias introduced due to measurement error in the marks due to HIV evolution after infection before sequence sampling (Sun and Gilbert, 2012). Dealing with this kind of bias is beyond the scope of this manuscript.

Goodness-of-fit tests of model (16) for each of the 10 marks were conducted using the testing procedure developed in Section 2. The test $T_{s}$ is based on the test statistic with $g_{k}\left(Z_{k i}, z\right)=$ $Z_{k i}$ and $T_{o}$ is based on $g_{k}\left(Z_{k i}, z\right)=I\left(Z_{k i} \leq z\right)$ with $\mathcal{C}=[0, \tau] \times[0.1,0.9] \times\{0,1\}$. Since $Z_{k i}$ is a Bernoulli variable, $T_{o}$ can be shown to be equal to $T_{s}$. The p-value for each mark is calculated based on 500 Gaussian multiplier simulations. Table 3 shows the value of the test statistic $T_{s}$ and the corresponding $p$-value for each of the ten marks. Table 3 also includes the selected bandwidth obtained using the formula $h=5 \hat{\sigma}_{V} n^{-1 / 3}$. All of the $p$-values are nonsignificant $(>0.05)$, supporting adequacy of the model fit for each data set.

[Table 3 about here]

Our results are consistent with the original primary analysis results of the Step trial data (not involving mark data, Buchbinder et al., 2008) that suggested the vaccine may make people more vulnerable to acquiring HIV infection. Re-analyzing the data ignoring the mark data, we fit the stratified proportional hazards model $\lambda_{k}(t \mid z)=\lambda_{0 k}(t) \exp \{\beta z\}$, for $k=1$ and 2, with result that $\hat{\beta}=0.422$ with standard error 0.254 and 2 -sided $p$-value 0.095 . Figure 2 shows the estimated survival functions for the vaccine and placebo groups stratified by pre-existing immunity to the Ad5 vector, showing a higher infection rate in the vaccine than placebo group within each Ad5 titer subgroup. 
[Figure 2 about here]

\section{Appendix}

Let $s_{k}^{(j)}(t, \beta)=E S_{k}^{(j)}(t, \beta)$, for $j=0,1,2$, and $J_{k}(t, \beta)=s_{k}^{(2)}(t, \beta) / s_{k}^{(0)}(t, \beta)-\left(s_{k}^{(1)}(t, \beta) /\right.$ $\left.s_{k}^{(0)}(t, \beta)\right)^{\otimes 2}$. A multivariate function is block-decreasing if it is decreasing in each variable. This definition is used in the condition (A.6). We make use of the following regularity conditions.

\section{Condition A}

(A.1) $\beta(v)$ has componentwise continuous second derivatives on $[0,1]$. For each $k=1, \ldots, K$, the second partial derivative of $\lambda_{0 k}(t, v)$ with respect to $v$ exists and is continuous on $[0, \tau] \times[0,1]$. The covariate process $Z_{k}(t)$ is left continuous with bounded variation, and satisfies the moment condition $E\left[\left\|Z_{k}(t)\right\|^{4} \exp \left(2 M\left\|Z_{k}(t)\right\|\right)\right]<\infty$, where $M$ is a positive constant such that $(v, \beta(v)) \in[0,1] \times(-M, M)^{p}$ for all $v$ and $\|A\|=\max _{k, l}\left|a_{k l}\right|$ for a matrix $A=\left(a_{k l}\right)$.

(A.2) Each component of $s_{k}^{(j)}(t, \theta)$ is continuous on $[0, \tau] \times[-M, M]^{p}$, and $\sup _{t \in[0, \tau], \theta \in[-M, M]^{p}}\left\|S^{(j)}(t, \theta)-s^{(j)}(t, \theta)\right\|=O_{p}\left(n^{-1 / 2}\right)$, for $j=0,1,2$.

(A.3) $s_{k}^{(0)}(t, \theta)>0$ on $[0, \tau] \times[-M, M]^{p}$, the limit $n_{k} / n \rightarrow p_{k}$ exists as $n \rightarrow \infty$ for $0<p_{k}<$ 1 and $1 \leq k \leq K$. The matrix $\Sigma(v)=\sum_{k=1}^{K} p_{k} \int_{0}^{\tau} J_{k}(t, \beta(v)) \lambda_{0 k}(t, v) s_{k}^{(0)}(t, \beta(v)) d t$ is positive definite for $v \in[0,1]$.

(A.4) $E\left(N_{k i}(d t, d v) \mid \mathcal{F}_{t-}\right)=E\left(N_{k i}(d t, d v) \mid Y_{k i}(t), Z_{k i}(t)\right)$, where $\left(\mathcal{F}_{t}\right)$ is the right-continuous filtration generated by the processes $\left(N_{k i}(t), Y_{k i}(t), Z_{k i}(t)\right), i=1, \ldots, n_{k}, k=1, \ldots, K$.

(A.5) The kernel function $K(\cdot)$ is symmetric with support $[-1,1]$ and of bounded variation, while the bandwidth satisfies $n h^{2} \rightarrow \infty$ and $n h^{4} \rightarrow 0$ as $n \rightarrow \infty$. 
(A.6) For each $k=1, \ldots, K$, the function $g_{k}(x, z)$ has the decomposition $g_{k}(x, z)=g_{k}^{+}(x, z)-$ $g_{k}^{-}(x, z)$, where $g_{k}^{+}(x, z)$ and $g_{k}^{-}(x, z)$ are two bounded nonnegative block-decreasing functions in $z$. For any $\epsilon>0$ there exists a $B>0$ such that (1) for $z_{1}, z_{2} \in[-B, B]^{p}$, $E\left\|g_{k}^{+}\left(Z_{k i}(t), z_{2}\right)-g_{k}^{+}\left(Z_{k i}(t), z_{1}\right)\right\|^{4} \leq C\left\|z_{2}-z_{1}\right\|$ and $E\left\|g_{k}^{-}\left(Z_{k i}(t), z_{2}\right)-g_{k}^{-}\left(Z_{k i}(t), z_{1}\right)\right\|^{4}$ $\leq C\left\|z_{2}-z_{1}\right\|$; (2) if for some $j=1, \ldots, p, z_{j, 1} \leq-B$ and $z_{j, 2} \leq-B$ or if $z_{j, 1} \geq B$ and $z_{j, 2} \geq B$, where $z_{j, 1}$ and $z_{j, 2}$ are respectively the $j$ th components of $z_{1}$ and $z_{2}$, then $E\left\|g_{k}^{+}\left(Z_{k i}(t), z_{2}\right)-g_{k}^{+}\left(Z_{k i}(t), z_{1}\right)\right\|^{4} \leq C \epsilon$ and $E\left\|g_{k}^{-}\left(Z_{k i}(t), z_{2}\right)-g_{k}^{-}\left(Z_{k i}(t), z_{1}\right)\right\|^{4} \leq$ $C \epsilon$, where $C$ is a constant independent of $t, z_{1}, z_{2}$ and $\epsilon$, and $\|\cdot\|$ is the Euclidean norm.

\section{Acknowledgements}

The authors thank the reviewers for their valuable inputs that have improved the paper. This research was partially supported by the National Institutes of Health NIAID [grant number R37 AI054165]. The research of Yanqing Sun was also partially supported by the National Science Foundation [grant number DMS-1208978] and funds provided by the University of North Carolina at Charlotte. We thank the HIV Vaccine Trials Network (HVTN) and Merck for providing the data analyzed in this article. The HVTN is supported through a cooperative agreement with the National Institutes of Health Division of AIDS, grant 5 U01 AI068635. The content is solely the responsibility of the authors and does not necessarily represent the official views of the National Institutes of Health.

\section{REFERENCES}

Aalen, O. O. and Johansen, S. (1978). An empirical transition matrix for non-homogeneous Markov chains based on censored observations. Scandinavian Journal of Statistics, 5, $141-150$.

Buchbinder, S. P., Mehrotra, D. V., and Duerr, A. et al. (2008). Efficacy assessment of a cell-mediated immunity HIV-1 vaccine (the Step Study): A double-blind, randomised, placebo-controlled, test-of-concept trial. Lancet, 372, 1881-1893. 
Buus, S., Lauemoller, S. L., Worning, P., et al. (2003). Sensitive quantitative predictions of peptide-MHC binding by a 'Query by Committee' artificial neural network approach. Tissue Antigens, 62, 378-384.

Cramer, E. and Schmiedt, A. B. (2011). Progressively Type-II censored competing risks data from Lomax distributions. Computational Statistics \& Data Analysis, 55, 1285-1303.

Feizjavadian, S. H. and Hashemi, R. (2015). Analysis of dependent competing risks in the presence of progressive hybrid censoring using Marshall-Olkin bivariate Weibull distribution. Computational Statistics \& Data Analysis, 82, 19-34.

Gilbert, P.B., Lele, S.R., Vardi, Y. (1999). Maximum likelihood estimation in semiparametric selection bias models with application to AIDS vaccine trials. Biometrika, 86, 27-43.

Gilbert, P.B., McKeague, I.W., Sun, Y. (2004). Tests for comparing mark-specfic hazards and cumulative incidence functions. Lifetime Data Analysis, 10, 5-28.

Gilbert, P.B., McKeague, I.W., Sun, Y. (2008). The two-sample problem for failure rates depending on a continuous mark: an application to vaccine efficacy. Biostatistics, 9, 263276.

Gilbert, P.B. and Sun, Y. (2014). Inferences on relative failure rates in stratified mark-specific proportional hazards models with missing marks, with application to HIV vaccine efficacy trials. Journal of the Royal Statistical Society: Series C, Early View, DOI: 10.1111/rssc.12067.

Han, D. and Balakrishnan, N. (2010). Inference for a simple step-stress model with competing risks for failure from the exponential distribution under time constraint. Computational Statistics \& Data Analysis, 54, 2066-2081.

Heckerman, D., Kadie, C., and Listgarten, J. (2007). Leveraging information across HLA alleles/supertypes improves epitope prediction. Journal of Computational Biology, 14, 736-46.

Huang, Y. and Louis, T.A. (1998). Nonparametric estimation of the joint distribution of survival time and mark variables. Biometrika, 85, 785-798. 
Lagakos, S. W. and Schoenfeld, D. A. (1984). Properties of proportional-hazards score tests under misspecified regression models. Biometrics, 40, 1037-1048

Lagakos, S. W. (1988b). Effects of mismodeling and mismeasuring explanatory variables on tests of association with a response variable. Statistics in Medicine, 7, 257-274.

Lin, D. Y., and Wei, L. J. (1989). The robust inference for the Cox proportional hazards model. Journal of the American Statistical Association, 84, 1074-1078.

Lin, D.Y., Wei, L.J, and Z.Ying. (1993) Checking the Cox model with cumulative sums of martingale-based residuals. Biometrika, 80, 557-572.

Olschewski, M. and Schumacher, M. (1990). Statistical analysis of quality of life in cancer clinical trials. Statistics in Medicine, 9, 749-763.

Rerks-Ngarm, S., Pitisuttithum, P., Nitayaphan, S., Kaewkungwal, J., Chiu, J., Paris, R., Premsri, N., Namwat, C., de Souza, M., Adams, E., Benenson, M., Gurunathan, S., Tartaglia, J., McNeil, J.G., Francis, D.P., Stablein, D., Birx, D.L., Chunsuttiwat, S., Khamboonruang, C., Thongcharoen, P., Robb, M.L., Michael, N.L., Kunasol, P., Kim, J.H. and the MOPHTAVEG Investigators. (2009). Vaccination with ALVAC and AIDSVAX to prevent HIV-1 infection in Thailand. The New England Journal of Medicine, 361, 2209-2220.

Struthers, C. and Kalbfleisch, J. D. (1986). Misspecified proportional hazards model. Biometrika, 74, 363-369.

Sun, Y., Gilbert, P. B. and McKeague, I. W. (2009). Proportional hazards models with continuous marks. The Annals of Statistics, 37, 394-426.

Sun, Y. and Gilbert, P. B. (2012). Estimation of stratified mark-specific proportional hazards models with missing marks. Scandinavian Journal of Statistics, 39, 34-52.

Sun, Y., Li, M. and Gilbert, P. B. (2013). Mark-specific proportional hazards model with multivariate continuous marks and its application to HIV vaccine efficacy trials. Biostatistics, 14, 60-74. 
Sun, Y. and Wu, H. (2005). Semiparametric time-varying coefficients regression model for longitudinal data. Scandinavian Journal of Statistics, 32, 21-47.

Zhou, H. and Wang, C.-Y. (2000) Failure time regression with continuous covariates measured with error. Journal Royal Statistical Society B, 62, 657-665. 
Table 1: Empirical sizes of the goodness-of-fit tests under models (11) and (12) at significant level 0.05. The test $T_{s}$ is based on the test statistic with $g_{k}\left(Z_{k i}, z\right)=Z_{k i}, T_{o}$ is based on $g_{k}\left(Z_{k i}, z\right)=I\left(Z_{k i} \leq z\right)$ and $T_{a}=T_{s}+T_{o}$. Each entry of the table is based on 500 simulations and 500 Gaussian multiplier samples. The bandwidth is selected using $h=3 \hat{\sigma}_{V} h^{-1 / 3}$.

\begin{tabular}{ccccc}
\hline \hline Model & $n$ & $T_{s}$ & $T_{o}$ & $T_{a}$ \\
\hline$M_{01}$ & 300 & 0.068 & 0.040 & 0.058 \\
& 400 & 0.050 & 0.052 & 0.044 \\
$M_{02}$ & 300 & 0.078 & 0.044 & 0.064 \\
& 400 & 0.052 & 0.046 & 0.058 \\
$M_{03}$ & 300 & 0.070 & 0.044 & 0.064 \\
& 400 & 0.058 & 0.052 & 0.048 \\
$M_{04}$ & 300 & 0.064 & 0.042 & 0.058 \\
& 400 & 0.062 & 0.060 & 0.056 \\
$M_{05}$ & 300 & 0.084 & 0.052 & 0.060 \\
& 400 & 0.068 & 0.058 & 0.064 \\
\hline
\end{tabular}


Table 2: Empirical powers of the goodness-of-fit tests under models (13), (14) and (15) at significant level 0.05. The test $T_{s}$ is based on the test statistic with $g_{k}\left(Z_{k i}, z\right)=Z_{k i}, T_{o}$ is based on $g_{k}\left(Z_{k i}, z\right)=I\left(Z_{k i} \leq z\right)$ and $T_{a}=T_{s}+T_{o}$. Each entry of the table is based on 500 simulations and 500 Gaussian multiplier samples. The bandwidth is selected using $h=3 \hat{\sigma}_{V} h^{-1 / 3}$.

\begin{tabular}{|c|c|c|c|c|c|}
\hline Model & Parameter & $n$ & $T_{s}$ & $T_{o}$ & $T_{a}$ \\
\hline \multicolumn{6}{|c|}{ Time varying alternative model (13) } \\
\hline \multirow[t]{2}{*}{$M_{11}$} & $\theta=2.0$ & 300 & 0.702 & 0.382 & 0.580 \\
\hline & & 400 & 0.766 & 0.462 & 0.672 \\
\hline \multirow[t]{2}{*}{$M_{12}$} & $\theta=2.5$ & 300 & 0.804 & 0.470 & 0.714 \\
\hline & & 400 & 0.878 & 0.576 & 0.788 \\
\hline \multirow[t]{2}{*}{$M_{13}$} & $\theta=3.0$ & 300 & 0.894 & 0.582 & 0.804 \\
\hline & & 400 & 0.946 & 0.694 & 0.866 \\
\hline \multicolumn{6}{|c|}{ Link function alternative model (14) } \\
\hline \multirow[t]{2}{*}{$M_{23}$} & $\alpha=0.15$ & 300 & 0.184 & 0.548 & 0.508 \\
\hline & & 400 & 0.222 & 0.694 & 0.638 \\
\hline \multirow[t]{2}{*}{$M_{22}$} & $\alpha=0.20$ & 300 & 0.166 & 0.574 & 0.534 \\
\hline & & 400 & 0.220 & 0.714 & 0.670 \\
\hline \multirow[t]{2}{*}{$M_{21}$} & $\alpha=0.25$ & 300 & 0.188 & 0.600 & 0.552 \\
\hline & & 400 & 0.224 & 0.746 & 0.704 \\
\hline \multirow{3}{*}{$M_{31}$} & Covariate & rm al & hative $n$ & lel (15) & \\
\hline & $\gamma=3.5$ & 300 & 0.074 & 0.824 & 0.762 \\
\hline & & 400 & 0.064 & 0.948 & 0.896 \\
\hline \multirow[t]{2}{*}{$M_{32}$} & $\gamma=4.0$ & 300 & 0.086 & 0.908 & 0.842 \\
\hline & & 400 & 0.058 & 0.976 & 0.944 \\
\hline \multirow[t]{2}{*}{$M_{33}$} & $\gamma=4.5$ & 300 & 0.090 & 0.944 & 0.910 \\
\hline & & 400 & 0.059 & 0.986 & 0.976 \\
\hline
\end{tabular}


Table 3: P-values of the goodness-of-fit test of model (16) under each of the 10 marks using the estimated bandwidth $h=5 \hat{\sigma}_{V} n^{-1 / 3}$. Each mark is identified by three components: Method, Ref and Protein. Method refers to the bioinformatics method (NetMHC or Epipred) used for the calculating the HIV sequence distances, Ref is for the reference HIV strain (the MRKAd5 vaccine sequence or the central HXB2 strain), and Protein is one of the four HIV-1 proteins (Gag, Pol, Nef and Gag-Pol-Nef) that are contained in the MRKAd5 vaccine and one additional set of proteins (Env-Rev-Tat-Vif-Vpr-Vpu) that is not contained in the MRKAd5 vaccine, serving as the control.

\begin{tabular}{|c|c|c|c|c|c|}
\hline \multicolumn{3}{|c|}{ Mark } & \multicolumn{3}{|c|}{ Goodness-of-Fit } \\
\hline Method & Ref & Protein & Estimated $h$ & Statistic $T_{s}$ & $p$-value \\
\hline Epipred & MRKAd5 & Gag & 0.360 & 0.107 & 0.108 \\
\hline Epipred & MRKAd5 & Pol & 0.336 & 0.065 & 0.744 \\
\hline Epipred & MRKAd5 & Nef & 0.375 & 0.054 & 0.844 \\
\hline Epipred & MRKAd5 & Gag-Pol-Nef & 0.333 & 0.082 & 0.474 \\
\hline Epipred & HXB2 & Env-Rev-Tat-Vif-Vpr-Vpu & 0.370 & 0.087 & 0.292 \\
\hline NetMHC & MRKAd5 & Gag & 0.350 & 0.121 & 0.968 \\
\hline NetMHC & MRKAd5 & Pol & 0.347 & 0.095 & 0.248 \\
\hline NetMHC & MRKAd5 & Nef & 0.324 & 0.079 & 0.180 \\
\hline NetMHC & MRKAd5 & Gag-Pol-Nef & 0.367 & 0.117 & 0.070 \\
\hline NetMHC & HXB2 & Env-Rev-Tat-Vif-Vpr-Vpu & 0.353 & 0.101 & 0.140 \\
\hline
\end{tabular}



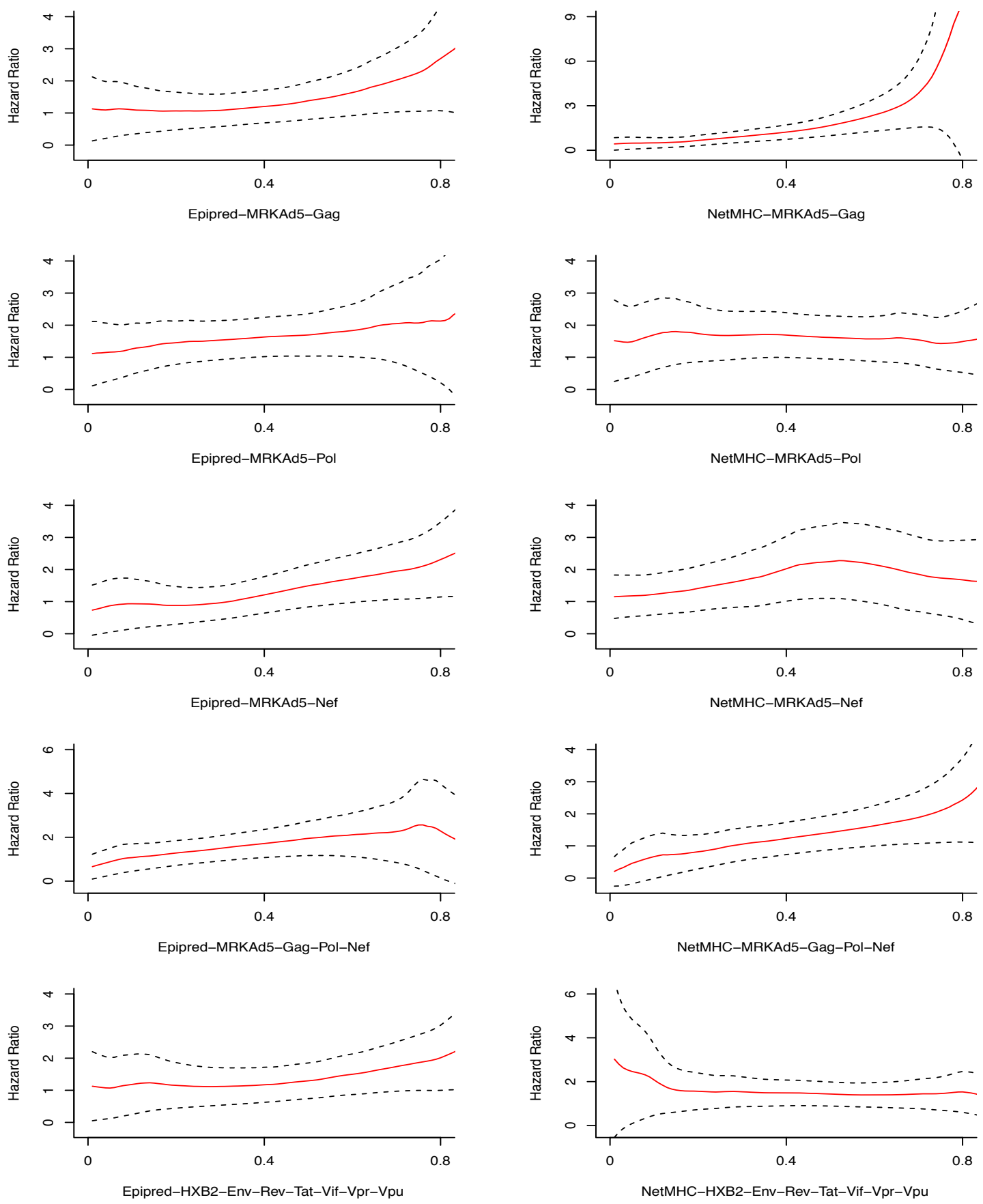

Figure 1: The plot of the estimated mark-specific hazard ratio $\exp \{\hat{\beta}(v)\}$ of the vaccine group versus the placebo group against the mark $v$ along with its $95 \%$ pointwise confidence intervals for each of the ten marks using the estimated bandwidth $h=5 \hat{\sigma}_{V} n^{-1 / 3}$. 


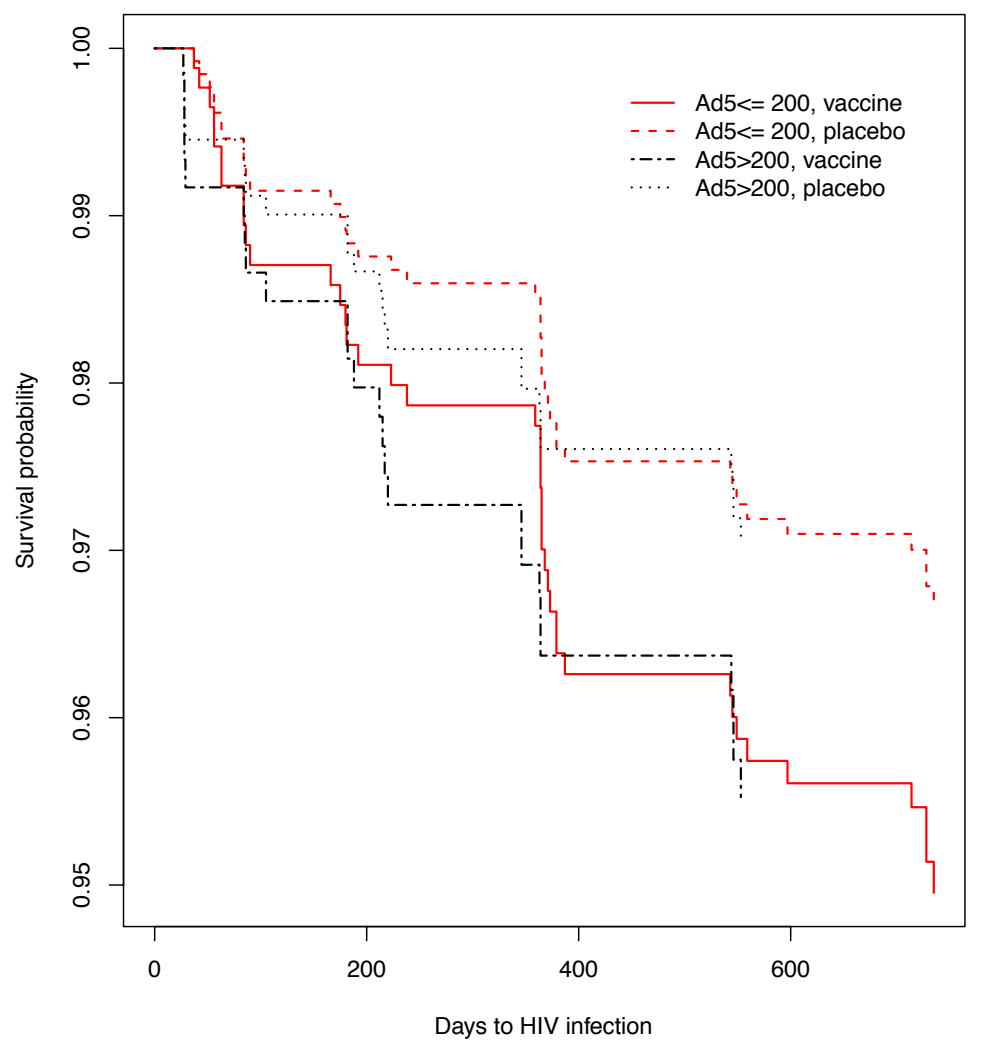

Figure 2: Plots of the estimated survival functions for the four groups defined according to the vaccination status and whether the subjects have the Ad5 immunity less than or grater than 200. 